\title{
A Cyclostationary Analysis Applied to Detection and Diagnosis of Faults in Helicopter Gearboxes ${ }^{\star}$
}

\author{
Edgar Estupiñan ${ }^{1}$, Paul White $^{2}$, and César San Martin ${ }^{3,4}$ \\ ${ }^{1}$ Department of Mechanical Engineering, Universidad de Tarapacá \\ Casilla 6-D, Arica, Chile \\ eestupin@uta.cl \\ ${ }^{2}$ Institute of Sound and Vibration,University of Southampton \\ SO17-1BJ, Southampton - U.K. \\ prw@isvr.soton.ac.uk \\ ${ }^{3}$ Department of Electrical Engineering, Universidad de Concepción \\ Casilla 160-C, Concepción, Chile \\ cesanmartin@udec.cl \\ ${ }^{4}$ Department of Electrical Engineering, Universidad de La Frontera \\ Casilla 54-D, Temuco, Chile \\ csmarti@ufro.cl
}

\begin{abstract}
In several cases the vibration signals generated by rotating machines can be modeled as cyclostationary processes. A cyclostationary process is defined as a non-stationary process which has a periodic time variation in some of its statistics, and which can be characterized in terms of its order of periodicity. This study is focused on the use of cyclic spectral analysis, as a tool to analyze second-order periodicity signals (SOP), such as, those who are generated by either localized or distributed defects in bearings. Cyclic spectral analysis mainly consists of the estimation of the random aspects as well as the periodic behavior of a vibration signal, based on estimation of the spectral correlation density. The usefulness of cyclic spectral analysis for the condition monitoring of bearings, is demonstrated in this paper, through the analysis of several sections of vibration data collected during an endurance test of one of the two main gearbox transmissions of a helicopter.
\end{abstract}

Keywords: Signal Processing, condition monitoring, vibration analysis, cyclostationarity.

\section{Introduction}

A cyclostationary process is a non-stationary process which has a periodic time variation in some of its statistics. The framework of cyclostationarity provides

\footnotetext{
* Most of this work was carried out at the ISVR of University of Southampton, funded with a grant from the Marie Curie Host Fellowships for Early Stage Research Training. The authors gratefully acknowledge the help of William Hardman who supplied the test bed data from H-60 tests conducted at the U. S. Navy's Helicopter Transmission Test Facility located at Patuxent River, Maryland and of Sally McInnery, University of Alabama.
} 
a powerful framework for modeling vibration signals from rotating machines. Such signals can be characterized by the different order of cyclostationarity they exhibit. In rotating machines, imbalances and misalignments can lead to vibrations that are examples of first-order periodicity processes (FOP). Whereas modulations generated by wear, friction forces or impact forces generate vibration signals that are second-order periodic (SOP) processes. To analyze FOP signals, different techniques such as the classical spectral analysis combined with time synchronous averaging can be employed. These methods provide powerful analysis tools suitable for many applications.

This study aims to consider the early detection faults in gearboxes, using vibration analysis, and exploiting the SOP structure of signals, through the use of cyclic spectral analysis. Cyclic spectral analysis involves the estimation of the random aspects of a signal as well as its periodic behavior. In this work the estimation of the spectral correlation density is computed using the averaged cyclic periodogram estimator 122 . The use of cyclostationarity for the detection and diagnosis of faults of bearings is demonstrated in this paper, through the analysis of vibration data registers from one of the two main gearboxes of an UH-60 Black Hawk helicopter.

This paper is organized as follows. In section 2 the cyclostationary analysis is presented. Section 3 presents the application of the cyclic spectral analysis to faults detection based on vibration signals processing. In section 4 we validate the proposed method using real vibration data register from gearboxes of an UH-60 Black Haw helicopter. In Section 5 the conclusions of the paper are summarized.

\section{Cyclostationary Analysis}

A cyclostationary process is considered as a stochastic process that exhibits some hidden periodicities, also called periodically correlated processes [3]. Nonstationary signals are considered cyclostationary when some of its statistics are periodic

$$
f_{x}(x, t)=f_{x}(x, t+T),
$$

where $f_{x}(x, t)$ denotes some appropriate, time varying, statistic of the signal. Some typical examples of cyclostationary signals are obtained through the periodic amplitude or frequency modulation of stationary processes.

A signal $x(t)$ is said to be $n^{t h}$ order cyclostationary with period $T$ if its $n^{\text {th }}$ order moments exist and are periodic with period $T$. A signal with firstorder periodicity is defined as one which has a finite-amplitude additive periodic component, and which consequently exhibits lines (Dirac delta functions) in its power spectral density. The FOP components can be separated from a signal through synchronous averaging. A pure FOP signal can be considered as being non-stationary in the sense that its mean is time-varying. Examples of FOP vibration signals that can be generated by rotating machines are imbalances, misalignments, anisotropic rotors, flexible coupling, etc. Some of the most common tools used to analyze (FOP) signals are: synchronous average, comb-filters, blind filters and adaptive comb-filters [4]. 
A signal with second-order periodicity (SOP) is defined as one which can be converted into a signal with FOP by a quadratic time invariant transformation 5]. These types of signals do not have a time-varying mean but do have a timevariant auto-correlation function. Stochastic processes with either amplitude or frequency modulation are typical examples of SOP signals. Faulty gearboxes may exhibit vibration signals that are amplitude modulated, leading to SOP, if, for example, the load being driven by the gearbox varies randomly.

Second order tools are based on the autocorrelation function. The instantaneous auto-correlation, the Wigner-Ville spectrum and the spectral correlation are second order tools, obtained from linear transformations of the autocorrelation function. For a cyclostationary signal $x(t)$, the auto-correlation function $(\mathrm{ACF})$ is defined by

$$
R_{x x}(t, \tau)=E\left\{x(t+\bar{\beta} \tau) x(t-\beta \tau)^{*}\right\},
$$

where $\bar{\beta}+\beta=1$. If $x(t)$ is cyclostationary with cycle (or period) $T$, then the $\mathrm{ACF}$ is also a cyclic function of time, i.e.,

$$
R_{x x}(t, \tau)=R_{x x}(t+T, \tau)
$$

and it can be expanded into their Fourier series and the Fourier coefficients of the ACF correspond to the cyclic ACF (CACF) given by

$$
R_{x x}(\tau, \alpha)=\int R(t, \tau) e^{-j 2 \pi \alpha t} d t
$$

where $\alpha$ correspond to the cyclic frequencies. The CACF gives an indication of how much energy in the signal is due to cyclostationarity at each frequency $\alpha$. Note that for $\alpha=0$, the CACF yields the conventional auto-correlation function. The Fourier transform of the CACF is known as the cyclic power spectrum given by

$$
S_{x x}^{\alpha}(\alpha, f)=\int R_{x x}(\tau, \alpha) e^{-j 2 \pi f \tau} d \tau,
$$

and we can note that the spectral correlation is a continuous function in frequency $f$ and a discrete function in terms of the cyclic frequency $\alpha$. For the case $\alpha=0$, the cyclic spectrum reduces to the classical power spectrum or spectral density function (through the Wiener-Khinchin relation) [3].

In the next section we introduce the cyclostationary analysis applied to the gearboxes and bearings, showing the potential of this analysis tools of detection and diagnosis of fault using vibration signals.

\section{Cyclic Spectral Analysis Applied to Faults Detections}

In several cases the vibration signals generated by rotating machines can be modeled as cyclostationary processes. For instance, cyclic spectral analysis results in appropriate tool to provide a statistical description of the random aspects of a 
cyclostationary vibration signal, as well as a description of the periodic behavior. In this work, we focused on vibrational signals measured from two rotating machines: gearbox and rolling bearings.

The vibration signals measured from a gearbox typically exhibit cyclostationarity of second and higher-orders. However, usually these components normally have a negligible energy when compared to the strong periodic signal generated by the meshing of the teeth. For this reason, it is important to subtract the synchronous average of the signal (FOP components) before analyzing the SOP cyclostationarity [4]. Vibrations generated by gears are typically polycyclostationary, since many different periodicities and periodic modulations associated with several rotating parts may be present in the raw signal.

For the rolling bearings signals, they usually exhibit a second order cyclostationary behavior, with the presence of localized as well as distributed defects [6]. In the case of localized defects in rolling bearings, a series of impacts are produced whose rate of repetition depends on their location. However, these impacts are not precisely periodic due to random slippage on each rotation; to reflect this effect the process is more correctly referred to as quasi-cyclostationary [7]. Further, the amplitude of the impacts can be modulated by the rotations of the inner race, outer race or the cage.

In practice, digital signal processing algorithms are required to estimate the cyclic-statistics of a cyclostationary process. In this paper we use the Averaged Cyclic Periodogram (ACP), which is one of the most common estimators used to estimate the spectral correlation function, because of its high computational efficiency [12]. In the cyclic spectrum, the cyclic frequencies $\alpha$, are multiples of the reciprocal of the period of the cyclostationarity. The ACP is defined by the expression

$$
S_{x x}^{\alpha}(f, \alpha)=\frac{1}{K \Delta_{t}} \sum_{K}^{k=1} X_{N}^{(k)}(f+\bar{\beta} \alpha) X_{N}^{(k)}(f+\beta \alpha)^{*},
$$

where $X_{N}^{(k)}$ is the discrete time Fourier transform of the $k t h$ sequence. In order to mitigate the effect of cyclic leakage, an overlap between adjacent segments of the signal should be incorporated. When a Hanning or Hamming data window is used, the overlap should be $\geq 67 \%$, in order to minimize the presence of cyclic leakage [1].

The cyclic coherence function is a useful tool for analysis of cyclostationary signals, to determine the strength of the correlation between spectral components spaced apart by cyclic frequencies. The cyclic coherence function is normalized between 0 and 1 , similarly to the spectral coherence. The cyclic coherence function for a single signal can be calculated from:

$$
\Gamma_{x x}(f, \alpha)=\frac{S_{x x}(f, \alpha)}{\left[S_{x}^{0}(f+\bar{\beta} \alpha) S_{x}^{0}(f-\bar{\beta} \alpha)\right]^{1 / 2}} .
$$




\section{Detection Faults in Helicopter Gearbox Using Cyclic Spectral Analysis}

In this section, sixty two vibration data registers of one of the two main gearboxes of an UH-60 Black Hawk helicopter have been analyzed using second order tools to identify the presence of cyclostationarity. The UH-60 Black Hawk main gearbox transmission is a complex system, composed of different gear transmissions, as it is shown in figure 1. This study is focus on the analysis of a fault detected in the inboard roller bearing SB-2205, which supports the combining bevel pinion in one of the input modules (see figure 2a). A fault in this bearing is particularly challenging since it is located deep inside the gearbox and the background noise may hidden the spectral components produced by the presence of a fault. Besides, the vibratory signal is also affected by the periodical components produced by the gear transmissions.

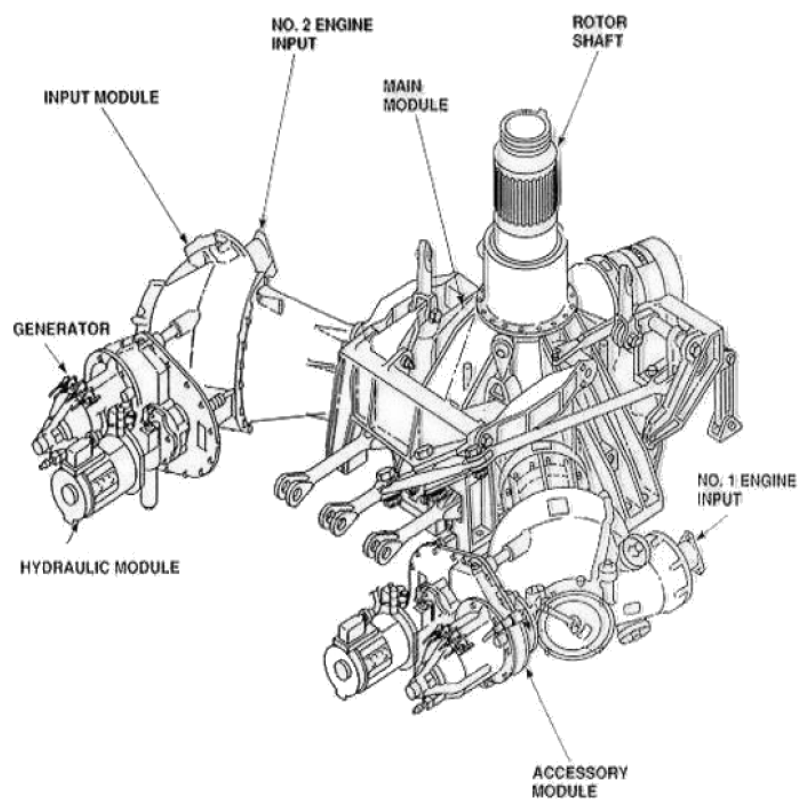

Fig. 1. Black Hawk's Main transmission

The vibration data were recorded during a component endurance test, carried out at Patuxent River, M.D. 8]. Accelerometers were used to acquire the data during the endurance test, at a rate of $100 \mathrm{kHz}$. Only the recordings within $\pm 10 \%$ of the full torque condition (sixty two data sets, each of 10 seconds duration), were used for this study. During this endurance test severe degradation of the inboard bearing SB-2205 occurred, and six chip lights were retrieved. The first gearbox chip light went on after 10200 minutes of run time had elapsed (which 
corresponds to the data set index No. 40). Figure $2 \mathrm{~b}$ shows a photograph of showing the final condition of the bearing rollers on completion of the endurance test.

A previous study analysing these vibration data was carried out by McInerny [9], which included the computation of power spectral densities and envelope spectra for selected frequency bands as well as trend plots of global indexes such as, deviation standard, kurtosis and wavelet coefficients. It was shown by McInerny that spectral components linked to the cage fault frequency (FTF) were identified in the envelope spectra for some frequency bands. It was also shown that the global indexes calculated showed an evident increase. However, spectral components directly related to the fault in the balls (ball spin frequencies, BSF), were not found.

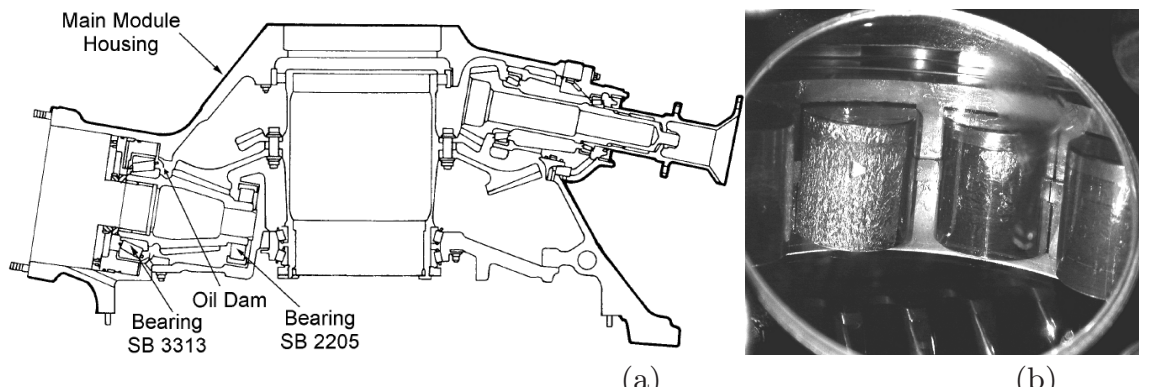

Fig. 2. a) Location of the SB-2205 bearing. b) Condition of the bearing rollers at the end of the endurance test.

In the present study, cyclic spectra and cyclic coherence functions were computed for all the sixty two vibration data registers recorded during the endurance test using equations (6) and (7). Before computing the cyclic spectra of the data, an adaptive strategy based on Adaptive Line Enhancer (ALE) was applied previously, to separate the FOP components and to focus the cyclostationary analysis on the residual signals [1011. To illustrate this process of filtering, Figure 3, shows the spectra of the residual signal (error signal, $e_{k}$ ) and the filter output $\left(y_{k}\right)$, after the ALE filter was applied to the vibration data register No. 42 (one of the data registers recorded after the first chip light went on).

To illustrate the results obtained with the cyclic spectral analysis, Figures 4 and 5, show the cyclic coherence function for the residual signal of data set registers No. 08 and No. 42 respectively. As it can be seen in figure 5, the cyclic coherence function revealed the existence of cyclic harmonics (hidden periodicities) linked with the ball spin frequency $(2 \times \mathrm{BSF}=361.5 \mathrm{~Hz})$ and the fault cage frequency $(\mathrm{FTF}=35.5 \mathrm{~Hz})$ of the inboard roller bearing. Besides, a detailed analysis of the cyclic spectrum let identified, sidebands spaced apart at $\alpha=35.5 \mathrm{~Hz}$ (FTF) around the harmonics at $1 \times \mathrm{BSF}$ and $2 \times \mathrm{BSF}$, indicating some degree of modulation. This cyclic harmonic structure is exactly similar to spectral harmonic structure ( $f$ domain) expected for a bearing with defects in the rollers [12]. 

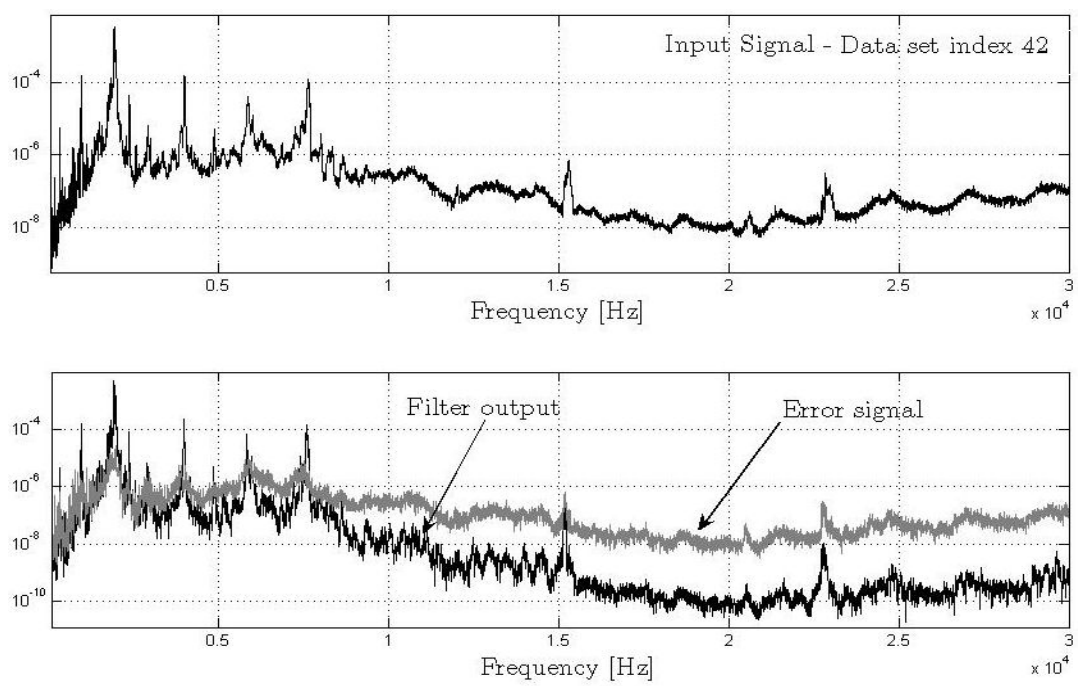

Fig. 3. Adaptive Line Enhancer (ALE), applied to one of the vibration data set, after the first chip light

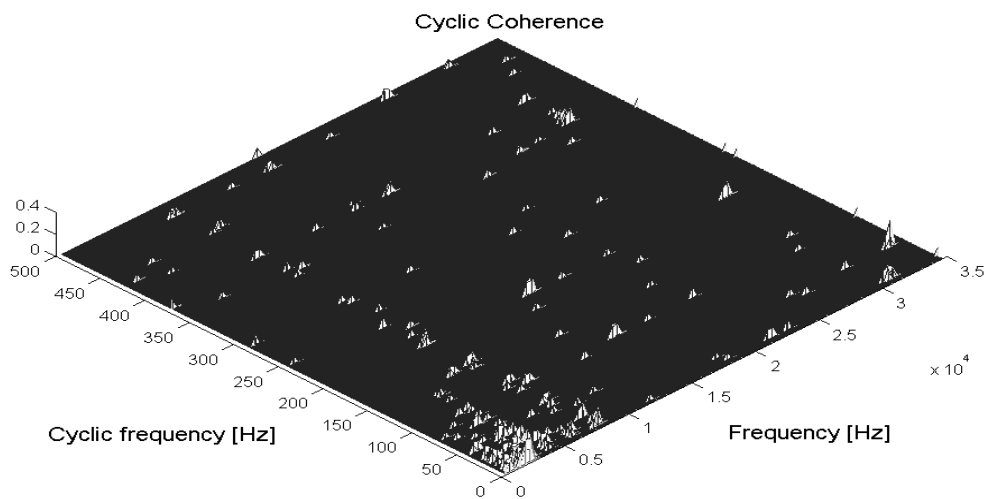

Fig. 4. Cyclic coherence function of fault-free case - Data set index 08. (Only values above $7.5 \%$ significance level are displayed).

It is important also to mention that these cyclic spectral components were not present in the cyclic coherence function when it was computed during the initial stages of the endurance test (see figure 4).

Additionally to the analysis of the spectral correlation functions, the cyclic spectra were compared for two different stages of fault, when they were computed at specific cyclic frequencies, linked to the fault bearing frequencies and the shaft rotational speed, as it is suggested in previous studies [213]. The results obtained are shown in figure 6. A considerable increase in the cyclostationary energy at the cyclic frequencies related to fault bearing frequencies was detected, as it can be seen in figures $6 \mathrm{~b}, 6 \mathrm{c}$ and $6 \mathrm{~d}$, which suggests that the cyclic spectra 


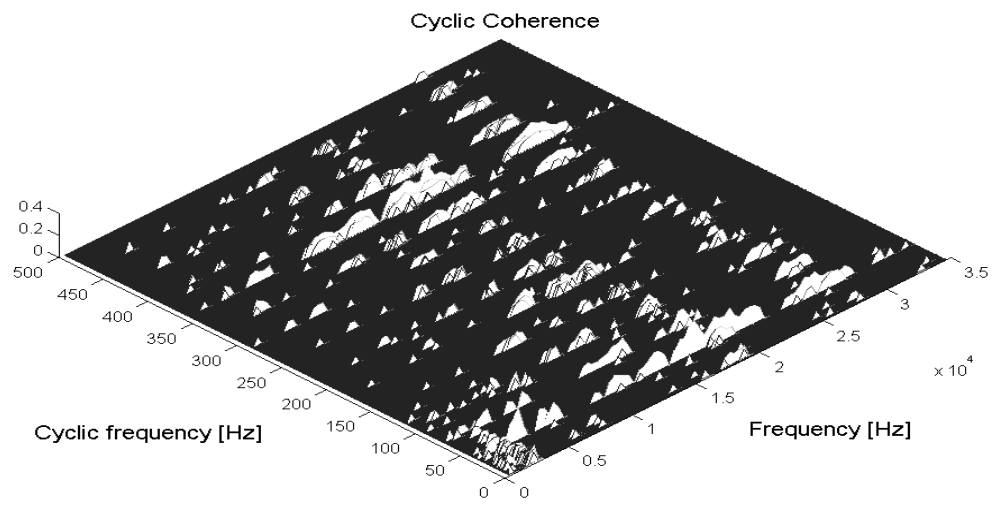

Fig. 5. Cyclic coherence function of faulty case - Data set index 42. (Only values above $7.5 \%$ significance level are displayed).
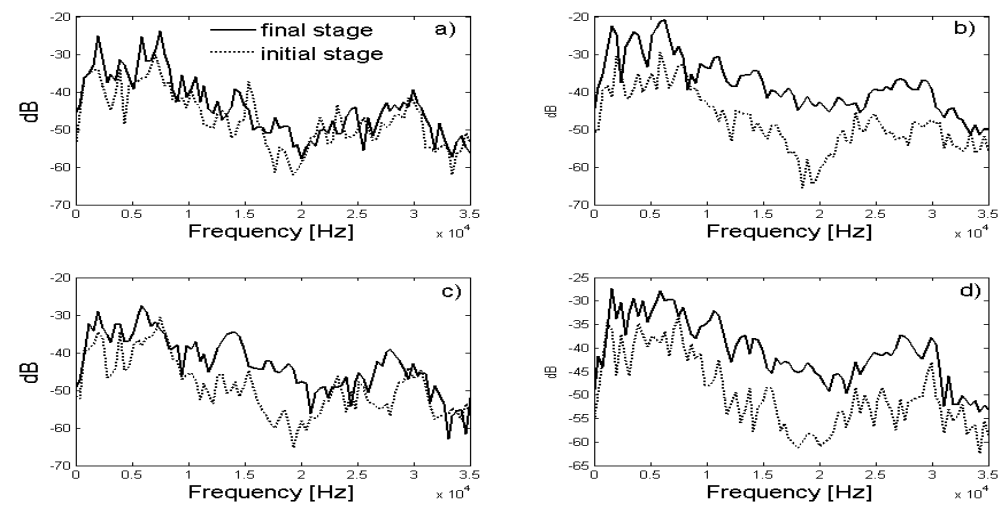

Fig. 6. Cyclic spectra computed at the cyclic frequencies: a) a $=95.5 \mathrm{~Hz}$ (shaft rotational speed), b) $a=35.5 \mathrm{~Hz}(1 \times \mathrm{xTF}), \mathrm{c}) \mathrm{a}=180.5 \mathrm{~Hz}(1 \mathrm{xBSF}), \mathrm{d}) \mathrm{a}=361.5 \mathrm{~Hz}(2 \mathrm{xBSF})$

computed at expected fault cyclic frequencies might be used as an indicator of damage intensity. Figure 6a, shows not evidence of increasing of the cyclostationary energy, indicating that the fault in this case is not related to the rotational frequency.

These last results suggest that the change of cyclostationarity should be not only analyzed for $\alpha=\Omega$ [1113] and better results could be obtained when the change of cyclostationairty is analyzed at cyclic frequencies related to the fault bearing frequencies. These results also confirm the SOP cyclostationary behavior of fault bearings, which has been demonstrated in [713. The SOP in fault bearings is caused mainly to the small randomness caused for the usual slip of the rolling elements and the cage. Therefore, the separation of vibrations produced by faults in gears (mainly in FOP components) from vibrations produced by faulty bearings (mainly SOP components) is a crucial aspect of the diagnosis of faults. 

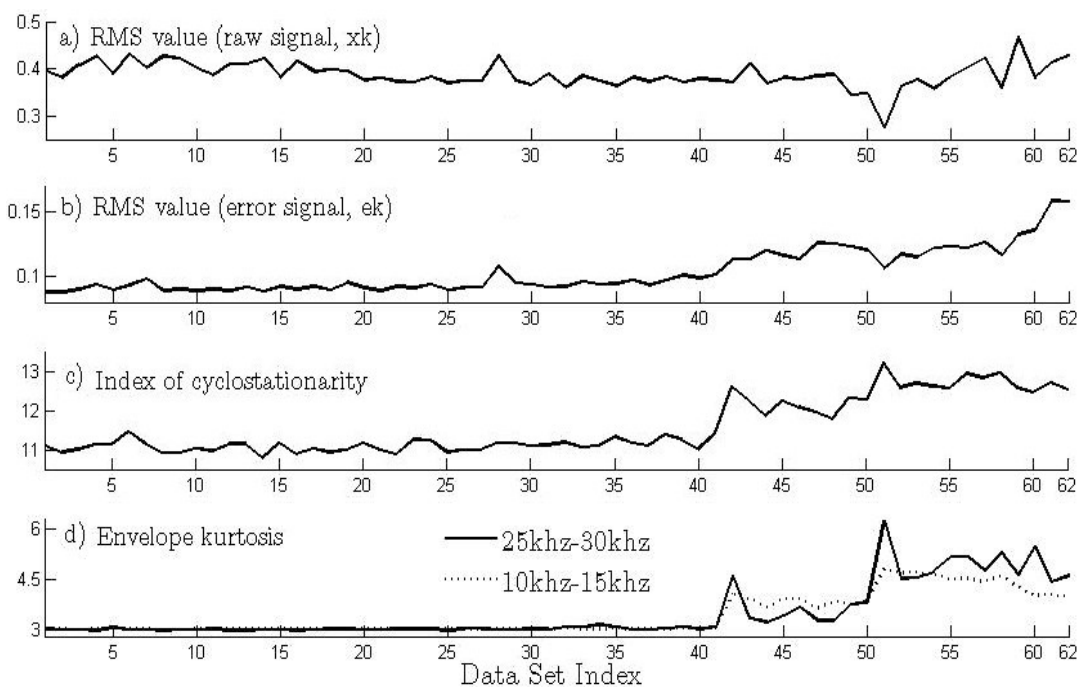

Fig. 7. Global indexes computed for all the vibration data sets

Finally, and to complement the cyclic spectral analysis, different global statistical metrics were computed for all of the data set indexes. It can be seen in figure 7 , that the RMS energy computed for the raw signals does not show evidence of great changes during all of the endurance tests. However, the other metrics shown demonstrate an evident increase, especially in the latter stages of the endurance tests, and after the first chip light. The index of cyclostationarity (8) and the envelope kurtosis (computed for two bandwidth frequencies) display the highest increase at the time when the first chip light went on, and only the RMS value computed for the error signal displays some transient increases in an early data set.

\section{Conclusions}

This study has demonstrated that cyclostationary analysis combined with an appropriate adaptive scheme, or another tool, to remove the FOP components from the signal, can be an efficiently tool to be applied to the vibration monitoring of rotating systems such as the gearbox of a helicopter.

This study has characterized properly the fault of the SB-2205 roller bearing produced during endurance tests of the UH-60A helicopter main gearbox. Through the computation of the cyclic coherence functions the fault frequencies of the bearing (cyclic spectral components at BSF), were detected, in contrast with a previous studies were they were not detected. Besides, an index of cyclostationarity is used as a global indicator and compared with the RMS global value, it demonstrates to have a better sensitivity to the presence of a fault. 
Nevertheless, further work should be focus on the testing of these indexes in more detail.

In this study the ACP was used as an estimator for the computation of the spectral correlation density, due to its high computational efficiency, however other estimators should be compared.

\section{References}

1. Boustani, R.: Sèparation aveugleà l'ordre deux de sources cyclostationnaires: application aux mesures vibroacoustiques. PhD Thesis, Universitè de Technologie Compiégne (2005)

2. Antoni, J.: Cyclic spectral analysis into practice. Mechanical Systems and Signal Processing 21(2), 597-630 (2007)

3. Gardner, W., Napolitano, A., Paura, L.: Cyclostationary: half a century of research. Signal Processing 86(4), 639-697 (2006)

4. Antoni, J., Bonnardot, F., Raad, A., El Badaoui, M.: Cyclostationary modelling of rotative machine vibration signals. Mechanical System and Signal Processing. 18(6), 1285-1314 (2004)

5. Gardner, W.: Statistical Spectral Analysis. Prentice-Hall, Englewood Cliffs (1991)

6. Bonnardot, F., Randall, R.B., Guillet, F.: Extraction of second- order cyclostationarity sources - Application to vibration analysis. Mechanical System and Signal Processing 19, 1230-1244 (2005)

7. Randall, B., Antoni, J., Chobsaard, S.: The relationship between spectral correlation and envelope analysis in the diagnostics of bearing faults and other cyclostationary machine signals. Mechanical Systems and Signal Processing 15(5), 945-962 (2001)

8. Dawson, F., Killian, K.: Alternate Source Endurance Qualification Test of UH-60 Black Hawk Transmission, Test Report. NAWCAD, Patuxent River, MD (June 2001)

9. McInerny, S.A., Hardman, B., Sun, Q.: Investigation of fault detection algorithms applied to a helicopter input pinion bearing. Technical Report (2004)

10. Lee, S., White, P.: The enhancement of impulsive noise and vibration signals for fault detection in rotating and reciprocating machinery. Journal of Sound and Vibration 217(3), 485-505 (1998)

11. Randall, R.B.: Detection and diagnosis of incipient bearing failure in helicopter gearboxes. Engineering Failure Analysis 11(2), 177-190 (2004)

12. McFadden, P.D., Smith, J.D.: Vibration monitoring of rolling element bearings by the high frequency resonance technique - a review. Tribology International 17(1) (1984)

13. Antoni, J., Randall, R.B.: Differential diagnosis of gear and bearing faults. Journal of Vibration and Acoustics 124(2), 1-7 (2002) 